\title{
The Resolved Kennicutt-Schmidt Law in Nearby Galaxies
}

\author{
R. Momose ${ }^{1,2,3}$, J. Koda ${ }^{4}$, R. C. Kennicutt, Jr ${ }^{5}$, F. Egusa ${ }^{6}$, \\ S. K. Okumura ${ }^{3,7}$, D. Calzetti ${ }^{8}$, G. Liu ${ }^{8,9}$, J. Donovan Meyer ${ }^{4}$, \\ N. Z. Scoville ${ }^{10}$, T. Sawada ${ }^{11}$, N. Kuno ${ }^{12}$ \\ ${ }^{1}$ ICRR, University of Tokyo, 5-1-5 Kashiwa-no-Ha, Kashiwa City, Chiba, 277-8582, Japan; \\ email: momo.s.rieko@nao.ac.jp; \\ ${ }^{2}$ University of Tokyo; ${ }^{3} \mathrm{NAOJ} ;{ }^{4} \mathrm{SUNY} ;{ }^{5} \mathrm{IoA}$; University of Cambridge, ${ }^{6} \mathrm{JAXA} ;{ }^{7} \mathrm{Japan}$ \\ Women's University; ${ }^{8}$ UMASS; ${ }^{9} \mathrm{JHU} ;{ }^{10}$ Caltech; ${ }^{11}$ Joint ALMA Office; ${ }^{12}$ NRO, NAOJ
}

\begin{abstract}
The Kennicutt-Schmidt law (Schmidt 1959; Kennicutt 1998, hereafter K-S law) is a power law correlation between area averaged star formation rate $\left(\Sigma_{S F R}\right)$ and gas surface density $\left(\Sigma_{\text {gas }}\right)$. Despite its importance, the physics that underlie this correlation has remained unclear. The power law index, $N$, is a prime discriminator of the mechanisms that regulate star formation and form the K-S law (e.g. Leroy et al. 2008; Tan 2010). We present a study of the resolved K-S law for 10 nearby disk galaxies using our new $\mathrm{CO}(1-0)$ data at 750 and $500 \mathrm{pc}$ resolutions. The $\mathrm{CO}(1-0)$ line emission is established as a tracer of the molecular gas column density, and results in a super-linear correlation $(N=1.3$ and 1.8). We discuss the cause of the discrepancy between previous studies, and the mechanism of star formation indicated from our new results.
\end{abstract}

Keywords. stars: formation — galaxies: spiral — ISM: molecules

\section{Results and Discussions}

We derive the K-S law on 750 pc scale by employing a similar procedure as Bigiel et al. (2008, hearafer B08), but with $\mathrm{CO}(1-0)$. The index at this scale shows a super-linear correlation with $N=1.3 \pm 0.02$. Furthermore we verify the K-S law on a 500 pc scale. The diffuse emission from both $\mathrm{H} \alpha$ and $24 \mu \mathrm{m}$ subtraction is also examined on this scale as an example. The best fit linear regressions also result in a super-linear slope both without and with diffuse emission subtraction $(N=1.2 \pm 0.05$ and $N=1.8 \pm 0.09$, respectively).

Our result is different from B08 $(N \sim 1)$. We find that the discrepancy of the slope between our results and B08 is caused largely by the adopted molecular gas tracer, $\mathrm{CO}(1-$ $0)$ or $\mathrm{CO}(2-1)$. $\mathrm{CO}(2-1)$ has only a factor of $2-3$ higher $n_{\text {crit }}\left(n_{\text {crit }} \geqslant 10^{3} \mathrm{~cm}^{-3}\right)$ and level energy (equivalent temperature of $16.5 \mathrm{~K}$ ); and thus, is excited more when the density and/or temperature is higher than the typical (a few $100 \mathrm{~cm}^{-3} ; 10 \mathrm{~K}$ ) even just mildly. In fact, the ratio of observed $\mathrm{CO}(2-1)$ to $\mathrm{CO}(1-0)$ intensity $\left(R_{21 / 10}\right)$ varies systematically with star formation rate and gas surface density in M51 (Koda et al. 2012). CO(2-1) likely traces the denser and/or warmer gas, rather than the bulk molecular gas in galaxies.

\section{References}

Bigiel, F. et al. 2008, AJ, 136, 2846

Kennicutt, Jr., R. C., 1998, ARAA, 36, 189

Koda, J. et al. 2012, accepted ApJ

Leroy, A. K. et al. 2008, AJ, 136, 2782

Schmidt, M., 1959, ApJ, 129, 243

Tan, J. C., 2010, ApJ, 710, L88 